\title{
USO DE INSTRUMENTO DE SUPORTE À TOMADA DE DECISÃO PARA GESTÃO DOS RECURSOS HÍDRICOS NO ESTADO DE MINAS GERAIS
}

\author{
Luís Antônio Coimbra Borges \\ Professor adjunto IV, Departamento de Ciências Florestais \\ Universidade Federal de Lavras - UFLA \\ luis.borges@dcf.ufla.br
}

Ana Luisa Alves Cabral Mestrado em Engenharia Florestal Universidade Federal de Lavras - UFLA analuisa.cabral@outlook.com

José Edimar Vieira Costa Júnior

Doutorando em Engenharia Florestal Universidade Federal de Lavras - UFLA jevcjunior@gmail.com

Luiz Otávio Moras Filho Doutorado em Engenharia Florestal Universidade Federal de Lavras - UFLA lomf22@gmail.com

\begin{abstract}
RESUMO
Objetivou-se avaliar a aplicação da Política Nacional de Recursos Hídricos (PNRH) no estado de Minas Gerais, analisando a implementação dos seus instrumentos. Para tal, foram estabelecidos dois indicadores relacionados à implementação dos instrumentos da PNRH (composto por 5 verificadores) e sobre a atuação dos comitês de bacias hidrográficas (composto por 3 verificadores) em cada Unidade de Planejamento e Gestão de Recursos Hídricos (UPGRH). Cada verificador foi analisado em relação ao seu percentual de cumprimento. Também foram atribuídos pesos aos verificadores por meio do Processo Hierárquico Analítico. Com base nesses valores, foi possível calcular o Índice de Contribuição Efetiva dos instrumentos da PNRH no estado de Minas Gerais, estimado em cerca de $71 \%$. Apesar do índice ser considerado alto, ainda são insuficientes no estado a aprovação do enquadramento dos corpos d'água, a cobrança pelo uso da água e o funcionamento de agências de bacias hidrográficas. A metodologia aplicada no presente trabalho demonstrouse adequada para a avaliação dos critérios propostos, possibilitando, inclusive, a agregação de novos critérios para avaliação dos instrumentos de gestão da PNRH.
\end{abstract}

Palavras-chave: Controle ambiental. Política nacional de recursos hídricos. Planejamento ambiental.

\section{USE OF A DECISION-MAKING SUPPORT INSTRUMENT FOR THE MANAGEMENT OF WATER RESOURCES IN THE STATE OF MINAS GERAIS}

\begin{abstract}
The aim of this study was to evaluate the application of the National Water Resources Policy $(\mathrm{PNRH})$ in Minas Gerais state, analysing the implementation of its instruments. In order to achieve this, two indicators related to the implementation of the PNRH instruments (composed of five verifiers) and the performance of the river basin committees (composed of three verifiers) in each Planning and Management Unit for Water Resources (UPGRH) were established. Each verifier was analysed in relation to its compliance percentage. Weights were also assigned to verifiers through the Analytical Hierarchical Process. Based on these figures, it was possible to calculate the Effective Contribution Index of the PNRH instruments in the state of Minas Gerais, estimated at around $71 \%$. Although the index is considered high, the approval of water bodies classification, charging for water use, and operation of river basin agencies is still insufficient in the state. The methodology applied in the present study proved to be adequate for the evaluation of the proposed criteria, allowing, also, the aggregation of new criteria for the evaluation of PNRH management instruments.
\end{abstract}

Keywords: Environmental control. National water resources policy. Environmental planning.

$\begin{array}{lllll}\text { Caminhos de Geografia } & \text { Uberlândia } & \text { v. 20, n. } 72 & \text { Dez/2019 } & \text { p. 315-325 Página } 315\end{array}$


Uso de instrumento de suporte à tomada de decisão para gestão dos recursos hídricos no estado de Minas Gerais
Luís Antônio Coimbra Borges Ana Luisa Alves Cabral José Edimar Vieira Costa Júnior Luiz Otávio Moras Filho

\section{INTRODUÇÃO}

Os recursos hídricos oferecem uma série de serviços ecossistêmicos fundamentais para a manutenção do meio ambiente e da sadia qualidade de vida. A disponibilidade hídrica, seja em quantidade ou em qualidade, sofre influência direta das atividades antrópicas a montante na bacia hidrográfica, tais como, uso de água para irrigação, construção de barragens, lançamento de efluentes, entre outros (TAFT e EVERS, 2016).

Aliar o crescimento econômico à preservação dos recursos naturais, sobretudo os hídricos, é uma das maiores preocupações que perpassa o debate ambiental (ARRUDA e NETO, 2017). O desenvolvimento econômico e tecnológico, somado ao acelerado crescimento populacional, tem gerado uma série de conflitos aos recursos naturais, destacando-se o uso da água (EDUARDO et al., 2016).

Entre os mais importantes e indispensáveis serviços ambientais necessários para a população, estão os serviços hídricos, que consistem em serviços de abastecimento, saneamento e pluviais (KATKO e HUKKA, 2015). A água detém grande valor para os habitantes ribeirinhos, por exemplo, dependência direta e indireta da disponibilidade, da qualidade e quantidade das águas dos rios, que são, por sua vez, influenciadas pela precipitação, evaporação e, principalmente, por atividades antropogênicas.

Em um contexto de processos produtivos ligados à terra, a administração inadequada ou uso extremo dos recursos hídricos pode gerar conflitos de uso da água, comprometer a capacidade dos serviços ambientais e o bem-estar dos habitantes. Conflitos pelo uso de recursos hídricos não são fenômenos recentes. O Tribunal de Águas de Valência tem mais de 1000 anos de atuação e é uma das cortes jurídicas mais antigas ainda em andamento.

Gerir os recursos hídricos é um desafio bastante complicado de acordo com Weng, Huang e Li (2010), visto a influência de impactos diversos e complexos, como socioeconômicos, ambientais e humanos, bem como, fatores naturais imprevisíveis - regime hidrológico das bacias hidrográficas.

Diante dessa problemática das águas no globo como um todo, e no Brasil especificamente, sobreveio a aprovação de um expressivo conjunto de normas jurídicas nacionais e internacionais visando regular as atividades humanas relacionadas a esse bem, que passa a ser cada vez mais juridicamente tutelado.

No Brasil, a Constituição de 1988 foi um marco para a gestão das águas ao instituir o Sistema Nacional de Gerenciamento de Recursos Hídricos (SNGRH), o qual teve sua regulamentação em 1997, por meio da Lei no 9.433, chamada de Lei das Águas, que definiu a Política Nacional de Recursos Hídricos (PNRH) (BRASIL, 1997).

De acordo com Lemos e Junior (2015), a lei das águas se baseia em princípios, fundamentos e instrumentos que devem ser contemplados nos processos de gestão de recursos hídricos no país. Todavia, as aplicações desses instrumentos de gestão devem se aproximar a cada realidade e contexto local da bacia hidrográfica. Para tal, a regulação possui especificidade a cada estado da Federação de acordo com as políticas estaduais de recursos hídricos e, se mais específico, pelos planos diretores de recursos hídricos das bacias.

Nesse contexto, o estado de Minas Gerais tem como órgão oficial de gestão das águas o Instituto Mineiro de Gestão das Águas (IGAM), regulamentado pelo decreto 47.343/2018, que atribui ao mesmo a finalidade de aplicar os instrumentos de gestão das águas e da política estadual de recursos hídricos (MINAS GERAIS, 2014).

Várias são as ferramentas e métodos utilizados no planejamento e gestão de recursos hídricos, como instrumentos de suporte à tomada de decisão, orientadas à gestão contínua e integrada e ao uso racional desses recursos. O Processo Hierárquico Analítico (Analytic Hierarchy Process - AHP), por exemplo, é um método clássico criado por Thomas L. Saaty em meados da década de 70 , em que sua aplicação se justifica frente à complexidade da tomada de decisões, como no caso da avaliação da gestão dos recursos hídricos em Minas Gerais (BOAS, 2005). Para Saaty (1990), o problema de decisão pode ser geralmente decomposto em níveis hierárquicos, facilitando assim, sua compreensão e avaliação.

Desse modo, objetivou-se com este trabalho analisar os instrumentos da PNRH e sua implementação no estado de Minas Gerais.

\section{METODOLOGIA}

\section{DESCRIÇÃO DA ÁREA DE ESTUDO}

Para a realização deste trabalho foram analisados os instrumentos da PNRH e a implementação dos mesmos no estado de Minas Gerais. 
O estado de Minas Gerais está localizado na região sudeste do Brasil. Possui uma área de 586.519,727 $\mathrm{km}^{2}$, o que corresponde a aproximadamente $7 \%$ da área total do país. Possui 853 municípios e uma população estimada no ano de 2018 de 21.040 .662 habitantes (INSTITUTO BRASILEIRO DE GEOGRAFIA E ESTATÍSTICA, 2018).

Minas Gerais abriga importantes nascentes de rios federais, como o rio São Francisco e o Rio Grande. Trata-se de um estado com abundância em recursos hídricos, mas que também sofre com problemas de inviabilidade devido à poluição e má distribuição. O estado é dividido em 4 regiões hidrográficas, 17 bacias e 36 Unidades de Planejamento de Gestão de Recursos Hídricos (UPGRH). A fim de se conhecer a situação dos instrumentos de planejamento da PNRH foram avaliadas as 36 UPGRH existentes em Minas Gerais (Figura 1).

Figura 1 - Configuração das 36 Unidades de Planejamento de Gestão de Recursos Hídricos (UPGRH) no estado de Minas Gerais.

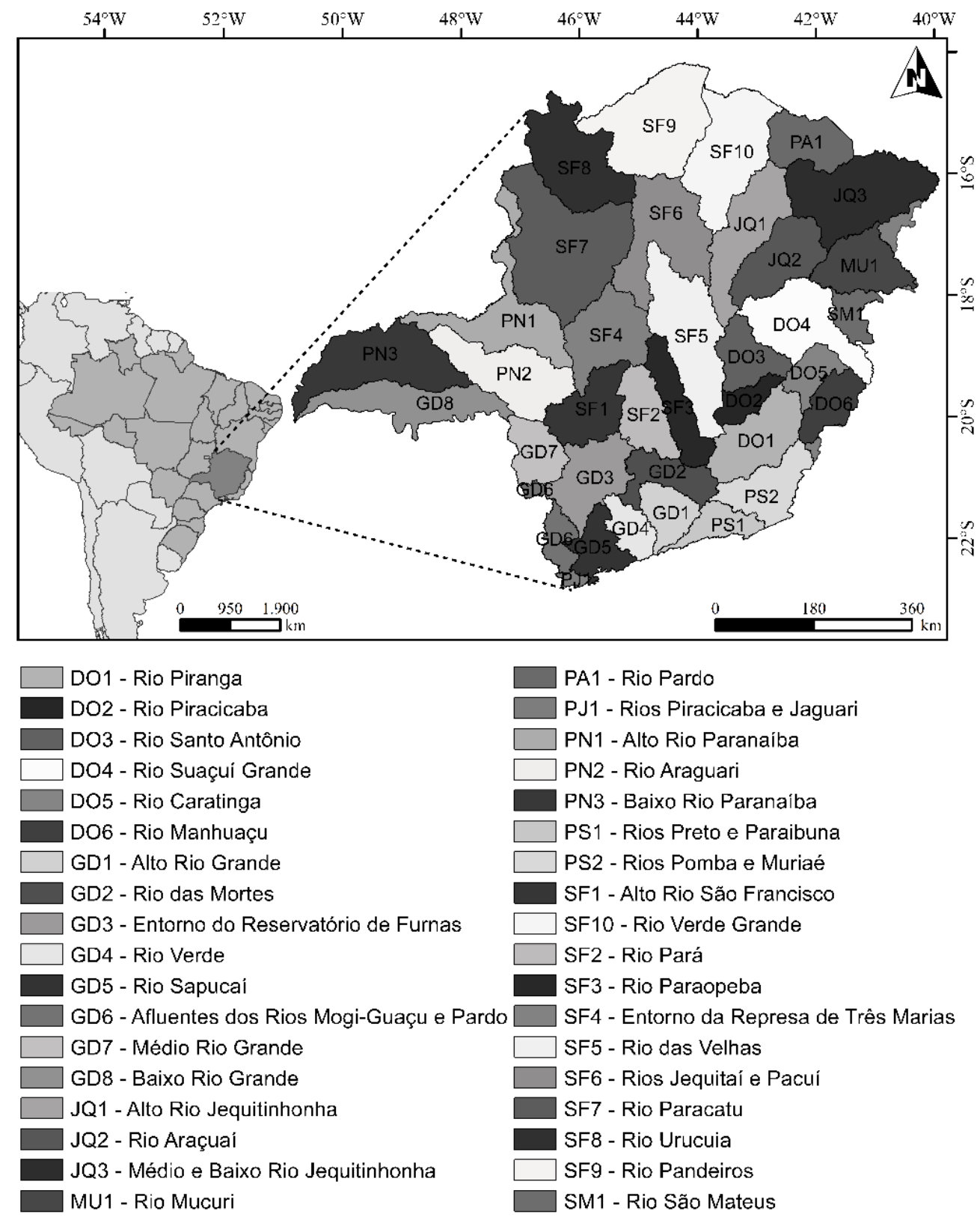

Fonte-es (2019).

$\begin{array}{llllll}\text { Caminhos de Geografia } & \text { Uberlândia } & \text { v. 20, n. } 72 & \text { Dez/2019 } & \text { p. 315-325 } & \text { Página } 317\end{array}$


Uso de instrumento de suporte à tomada de decisão para gestão dos recursos hídricos no estado de Minas Gerais
Luís Antônio Coimbra Borges Ana Luisa Alves Cabral José Edimar Vieira Costa Júnior Luiz Otávio Moras Filho

\section{SEQUÊNCIA METODOLÓGICA}

\section{PRIMEIRA ETAPA: PESQUISA BIBLIOGRÁFICA E DOCUMENTAL}

Foi realizada pesquisa bibliográfica e documental com a finalidade de levantar os estudos científicos já realizados sobre o assunto, as legislações que regem esses instrumentos e os relatórios oficiais sobre os mesmos. Segundo Selltiz, Wrightsman e Cook (1965), esse tipo de pesquisa busca descobrir ideias e intuições, na tentativa de adquirir maior familiaridade com o fenômeno pesquisado.

Como fonte de dados, buscou-se documentos jurídicos e institucionais, produções científicas e dados da gestão dos recursos hídricos em Minas Gerais obtidos em arquivos públicos disponíveis no sítio eletrônico do Sistema Estadual sobre os Recursos Hídricos do estado de Minas Gerais (InfoHidro).

O levantamento desses dados foi baseado em uma amostragem não probabilística do tipo acessibilidade. Conforme Alencar (2003) trata-se de um método que possibilita encontrar certo elemento em um universo desconhecido

\section{SEGUNDA ETAPA: DEFINIÇÃO DO PRINCÍPIO, CRITÉRIOS, INDICADORES E VERIFICADORES}

O Centro Internacional para Pesquisa Florestal (CIFOR, 1999) sugere que um Método de Multicritérios de Análise de Decisão (MMAD) deva seguir, de forma hierárquica, princípios, critérios, indicadores e verificadores, em que se entende por:

a) Princípio: verdade ou lei fundamental tomada como raciocínio ou ação, sendo que eles fornecem justificativas para os critérios, indicadores e verificadores.

b) Critérios: princípio ou padrão pelo qual alguma coisa é julgada. São pontos intermediários aos quais a informação fornecida pelos indicadores pode ser integrada e nos quais uma avaliação interpretável se cristaliza.

c) Indicadores: qualquer variável que pode ser medida com relação a um critério. Ainda pode-se inferir que indicadores são ferramentas constituídas por uma ou mais variáveis, que associadas por meio de diversas formas revelam significados mais amplos sobre os fenômenos a que se referem (INSTITUTO BRASILEIRO DE GEOGRAFIA E ESTATÍSTICA, 2004).

d) Verificadores: dados ou informações que aumentam a especificidade ou a facilidade de avaliação de um indicador. Eles fornecem os detalhes especiais que indicam ou refletem uma condição desejada de um indicador (MENDOZA et al., 1999).

Em linhas gerais, os princípios podem ser entendidos como metas a serem alcançadas e, junto com os critérios, apontam o "quê está sendo analisado", enquanto os indicadores e verificadores indicam "se e, até que ponto" a meta foi atendida (GOMES, 2000). Dessa maneira, foi elaborada uma hierarquia de decisão para este trabalho, conforme apresentado no Quadro 1.

Quadro 1 - Organização do princípio, critérios, indicadores e verificadores utilizados na avaliação da aplicação da Política Nacional de Recursos Hídricos no estado de Minas Gerais

\begin{tabular}{|c|c|}
\hline \multicolumn{2}{|c|}{ Princípio } \\
\hline $\begin{array}{l}\text { Os instrumentos de gestão da Política Nacional de } \\
\text { dos recursos hídricos no estado de Minas Gerais }\end{array}$ & ursos Hídricos contribuem para a gestão adequada \\
\hline Critério 1 & Critério 2 \\
\hline $\begin{array}{l}1 \text { A implementação dos instrumentos de gestão da } \\
\text { PNRH nas UPGRHs contribui para a gestão } \\
\text { adequada dos Recursos Hídricos em MG. }\end{array}$ & $\begin{array}{l}2 \text { A gestão nas UPGRHs contribui para a } \\
\text { manutenção dos recursos hídricos }\end{array}$ \\
\hline Indicadores & Indicadores \\
\hline 1.1 Implementação dos Instrumentos da PNRH & 2.1 Comitê atuante na UPGRH \\
\hline Verificadores & Verificadores \\
\hline $\begin{array}{l}\text { 1.1.1 Plano diretor de recursos hídricos aprovado } \\
\text { 1.1.2 Enquadramento dos corpos d'água aprovado } \\
\text { 1.1.3 Outorga de direito de uso da água } \\
\text { 1.1.4 Cobrança pelo uso da água } \\
\text { 1.1.5 Dados disponíveis no Sistema de Informações }\end{array}$ & $\begin{array}{l}\text { 2.1.1 Comitê de bacia em funcionamento } \\
\text { 2.1.2 Agência de bacia em funcionamento } \\
\text { 2.1.3 UPGRHs com comitês com mais de } 10 \text { anos }\end{array}$ \\
\hline
\end{tabular}

Fonte - adaptado de CIFOR (1999). 
Uso de instrumento de suporte à tomada de decisão para gestão dos recursos hídricos no estado de Minas Gerais
Luís Antônio Coimbra Borges Ana Luisa Alves Cabral José Edimar Vieira Costa Júnior Luiz Otávio Moras Filho

Buscando analisar a consistência do primeiro critério, foi estabelecido um indicador relacionado à implementação dos instrumentos da PNRH, composto por 5 verificadores. Foram acessadas no Sistema de Informações sobre Recursos Hídricos do estado de Minas Gerais, o Portal InfoHidro, as seguintes informações: UPGRHs cujo plano diretor de recursos hídricos (verificador 1.1.1) e enquadramento dos corpos d'água (verificador 1.1.2) já havia sido aprovado; outorgas deferidas no período de 2008 a 2013 e cadastros de uso insignificante realizados entre 2008 e 2012 para cada UPGRH (verificador 1.1.3); informações referentes às UPGRHs que já implementaram a cobrança pelo uso da água (verificador 1.1.4); e UPGRHs que já haviam disponibilizado dados no sistema de informações (verificador 1.1.5).

Já em relação ao segundo critério, foi definido um indicador referente ao grau de atuação do comitê gestor de cada UPGRH, composto por 3 verificadores. No Portal InfoHidro, foram acessadas para cada UPGRH informações sobre: o funcionamento do comitê de bacia (verificador 2.1.1) das agências de bacia (verificador 2.1.2), bem como se os comitês foram implementados há mais de 10 anos (verificador 2.1.3).

\section{TERCEIRA ETAPA: ELABORAÇÃO E NORMALIZAÇÃO DA MATRIZ DE JULGAMENTO}

Para elaborar a matriz de julgamento foi utilizada a técnica AHP por meio da escala fundamental de números absolutos conhecida por escala Saaty (2013), que busca comparar os verificadores em pares, gerando um valor numérico que varia de acordo com o exposto no Quadro 2.

Quadro 2 - Escala Numérica de Saaty.

\begin{tabular}{|c|c|c|}
\hline Escala numérica & Escala Verbal & Explicação \\
\hline 1 & Igual importância & $\begin{array}{l}\text { Ambos os itens (critérios ou alternativas) } \\
\text { contribuem igualmente para atingir o objetivo }\end{array}$ \\
\hline 3 & Importância moderada & $\begin{array}{l}\text { Experiência e julgamento favorecem } \\
\text { ligeiramente um item (critérios ou } \\
\text { alternativas) sobre o outro }\end{array}$ \\
\hline 5 & Forte importância & $\begin{array}{l}\text { Experiência e julgamento favorecem } \\
\text { fortemente um item sobre o outro }\end{array}$ \\
\hline 7 & Importância muito forte & $\begin{array}{l}\text { Um item é muito fortemente favorecido em } \\
\text { detrimento de outro; sua dominância é } \\
\text { demonstrada na prática }\end{array}$ \\
\hline 9 & $\begin{array}{l}\text { Importância absoluta ou extrema } \\
\text { importância }\end{array}$ & $\begin{array}{l}\text { A evidência favorecendo uma atividade } \\
\text { sobre a outra é da mais alta ordem possível } \\
\text { de afirmação }\end{array}$ \\
\hline $2,4,6$ e 8 & $\begin{array}{l}\text { Valores intermediários entre as } \\
\text { opiniões adjacentes. }\end{array}$ & $\begin{array}{l}\text { Usados como valores de consenso entre as } \\
\text { opiniões. }\end{array}$ \\
\hline Incremento $\mathbf{0 , 1}$ & $\begin{array}{l}\text { Valores intermediários na } \\
\text { graduação mais fina de } 0,1 .\end{array}$ & $\begin{array}{l}\text { Usados para graduações mais finas das } \\
\text { opiniões. }\end{array}$ \\
\hline Recíprocos & $\begin{array}{l}\text { Valores inversos aos pesos de } \\
\text { julgamentos: }(1 / n) \text { onde "n" é o } \\
\text { peso atribuído }\end{array}$ & Suposição lógica \\
\hline
\end{tabular}

Fonte - adaptado de Saaty (2013).

Após a construção da matriz, foi necessário realizar a normalização dos dados por meio do Índice de Consistência (IC) proposto por Mendoza (1999) por meio da seguinte equação:

Equação 1. $a_{i j}=a_{i j} \div \sum_{i=1}^{n} a_{i j}$

Onde: $a_{i j}=$ comparação na matriz "a" da linha "i" com a coluna "j", $\mathrm{n}=$ número de elementos.

\section{QUARTA ETAPA: VERIFICAÇÃO DO ÍNDICE E DA TAXA DE CONSISTÊNCIA}

A contribuição na meta organizacional de cada verificador é determinada a partir do autovetor (Eigenvector), ou vetor de prioridade média, que apresenta pesos relativos entre os verificadores e é obtido por meio da média aritmética dos valores de cada um dos verificadores (VARGAS, 2010). Após esse procedimento, foi 
calculado o número principal do autovetor que, segundo Godoi (2014), é obtido por meio da somatória do produto de cada elemento do autovetor, multiplicados pelo total de cada coluna calculada na Matriz de Julgamento.

O Índice de Consistência (IC) é uma medida de quão lógico os julgamentos do pesquisador são (MENDOZA et al., 1999), obtido por meio da seguinte equação:

Equação 2. $\quad \mathrm{IC}=\frac{\left(\lambda_{\max }-n\right)}{n-1}$

Onde: $\lambda_{\max }=$ maior autovetor (Eigenvector) da matriz, equivalente ao número principal do autovetor; $\mathrm{n}=$ número de verificadores da matriz.

Para se calcular a Taxa de Consistência (TC), e enfim, saber se existe coerência na matriz de julgamento, utiliza-se o Índice Randômico (IR) proposto por Saaty (2008), que varia de acordo com o número de verificadores (Tabela 9).

Tabela 1 - Índice Randômico Médio da AHP.

\begin{tabular}{llllllllllll}
\hline $\mathbf{N}$ & $\mathbf{0}$ & $\mathbf{1}$ & $\mathbf{2}$ & $\mathbf{3}$ & $\mathbf{4}$ & $\mathbf{5}$ & $\mathbf{6}$ & $\mathbf{7}$ & $\mathbf{8}$ & $\mathbf{9}$ & $\mathbf{1 0}$ \\
\hline $\mathbf{I R}$ & 0 & 0 & 0 & 0,58 & 0,9 & 1,12 & 1,24 & 1,32 & 1,41 & 1,45 & 1,49 \\
\hline
\end{tabular}

Fonte - adaptado de Saaty (2008).

A TC dos indicadores é calculada a partir da razão entre o IC e IR. A matriz será considerada consistente se a TC for menor que $10 \%$ (VARGAS, 2010).

\section{QUINTA ETAPA: CÁLCULO DA CONTRIBUIÇÃO DE CADA VERIFICADOR E CÁLCULO DO ÍNDICE DE CONTRIBUIÇÃO EFETIVA}

A cada verificador foi atribuído um peso referente ao valor principal de cada autovetor da matriz normalizada. Para verificar a contribuição de cada verificador, foi multiplicado esse peso pelo percentual de UPGRHs que cumpriram cada verificador.

Para o cálculo dos Índice de Contribuição Efetiva (ICE) foi feita a média aritmética do percentual de contribuição dos dois indicadores. Os valores de ICE foram enquadrados em 4 classes de contribuição, de acordo com a tabela 2.

Tabela 2 - Índice Classes de contribuição para cada faixa de variação do Índice de Contribuição Efetiva (ICE) dos instrumentos de gestão da Política Nacional de Recursos Hídricos no estado de Minas Gerais.

\begin{tabular}{cc}
\hline ICE & Contribuição \\
\hline 0 a $40 \%$ & Contribuição baixa \\
41 a $60 \%$ & Contribuição média \\
61 a $80 \%$ & Contribuição alta \\
81 a $100 \%$ & Contribuição muito alta \\
\hline
\end{tabular}

Fonte-es (2019).

\section{RESULTADOS E DISCUSSÃO}

De acordo com a matriz hierárquica definida neste trabalho, foi realizado um levantamento do cumprimento dos verificadores na implementação dos instrumentos da PNRH e da atuação dos comitês nas UPGRHs, de acordo com a Tabela 3. 
Tabela 3 - Cumprimento dos verificadores utilizados para avaliar instrumentos de gestão da Política Nacional de Recursos Hídricos no estado de Minas Gerais.

\begin{tabular}{lrr}
\hline Verificadores/indicador & $\begin{array}{r}\text { No de UPGRHs em } \\
\text { que o verificador } \\
\text { foi cumprido }\end{array}$ & $\begin{array}{r}\text { Percentual de UPGRHs } \\
\text { em que o verificador foi } \\
\text { cumprido (\%) }\end{array}$ \\
\hline 1.1 Implementação dos Instrumentos da PNRH & 27 & 75,00 \\
\hline 1.1.1 Plano diretor de recursos hídricos aprovado & 8 & 22,22 \\
1.1.2 Enquadramento dos corpos d'água aprovado & 36 & 100,00 \\
1.1.3 Outorga de direito de uso da água & 11 & 30,55 \\
1.1.4 Cobrança pelo uso da água & 36 & 100,00 \\
1.1.5 Dados disponíveis no Sistema de Informações & & 100,00 \\
\hline 2.1 Comitê atuante na UPGRH & 36 & 33,33 \\
\hline 2.1.1 Comitê de bacia em funcionamento & 12 & 72,22 \\
2.1.2 Agência de bacia em funcionamento & 26 & \\
2.1.3 UPGRHs com comitês com mais de 10 anos & & \\
\hline
\end{tabular}

Fonte-es (2019).

Foi também elaborada a matriz de julgamento de cada um dos verificadores com base na escala comparativa proposta por Saaty (2013), tanto para o indicador 1 (tabela 4) quanto para o indicador 2 (tabela 5).

Tabela 4 - Matriz de julgamento para o indicador 1.1.

\begin{tabular}{crrrrr}
\hline & Ver. 1.1.1 & Ver. 1.1.2 & Ver. 1.1.3 & Ver. 1.1.4 & Ver. 1.1.5 \\
\hline Ver. 1.1.1 & 1,000 & 3,000 & 3,000 & 5,000 & 7,000 \\
Ver. 1.1.2 & 0,333 & 1,000 & 5,000 & 3,000 & 2,000 \\
Ver. 1.1.3 & 0,333 & 0,200 & 1,000 & 2,000 & 2,000 \\
Ver. 1.1.4 & 0,200 & 0,333 & 0,500 & 1,000 & 2,000 \\
Ver. 1.1.5 & 0,143 & 0,500 & 0,500 & 0,500 & 1,000 \\
\hline Total & 2,009 & 5,033 & 10,000 & 11,500 & 14,000 \\
\hline
\end{tabular}

Fonte-es (2019).

Tabela 5 - Matriz de julgamento para o indicador 2.1.

\begin{tabular}{rrrr}
\hline & Ver. 2.1.1 & Ver. 2.1.2 & Ver. 2.1.3 \\
\hline Ver. 2.1.1 & 1,000 & 3,000 & 5,000 \\
Ver. 2.1.2 & 0,333 & 1,000 & 3,000 \\
Ver. 2.1.3 & 0,200 & 0,333 & 1,000 \\
\hline Total & 1,533 & 4,333 & 9,000 \\
\hline \multicolumn{4}{c}{ Fonte - es (2019). }
\end{tabular}

Os julgamentos paritários normalizados, visando a obtenção do Índice de Consistência, também foram obtidos tanto para o indicador 1 (tabela 6) quanto para o indicador 2 (tabela 7).

Tabela 6 - Matriz de julgamento normalizada para o indicador 1.1

\begin{tabular}{rrrrrr}
\hline & Ver. 1.1.1 & Ver. 1.1.2 & Ver. 1.1.3 & Ver. 1.1.4 & Ver. 1.1.5 \\
\hline Ver. 1.1.1 & 0,498 & 0,596 & 0,300 & 0,435 & 0,498 \\
Ver. 1.1.2 & 0,166 & 0,199 & 0,500 & 0,261 & 0,166 \\
Ver. 1.1.3 & 0,166 & 0,040 & 0,100 & 0,174 & 0,166 \\
Ver. 1.1.4 & 0,100 & 0,066 & 0,050 & 0,087 & 0,100 \\
Ver. 1.1.5 & 0,071 & 0,099 & 0,050 & 0,043 & 0,071 \\
\hline
\end{tabular}

Fonte - es (2019).

\begin{tabular}{|c|c|}
\hline & Uberlândia \\
\hline
\end{tabular}


Uso de instrumento de suporte à tomada de decisão para gestão dos recursos hídricos no estado de Minas Gerais
Luís Antônio Coimbra Borges Ana Luisa Alves Cabral José Edimar Vieira Costa Júnior Luiz Otávio Moras Filho

Tabela 7 - Matriz de julgamento normalizada para o indicador 2.1.

\begin{tabular}{rrrr}
\hline & Ver. 2.1.1 & Ver. 2.1.2 & Ver. 2.1.3 \\
\hline Ver. 2.1.1 & 0,65 & 0,69 & 0,56 \\
Ver. 2.1.2 & 0,22 & 0,23 & 0,33 \\
Ver. 2.1.3 & 0,13 & 0,08 & 0,11 \\
\hline
\end{tabular}

Fonte-es (2019).

Os autovetores (Eigenvectors), ou vetores de prioridade, que representam o peso relativo entre os verificadores foram calculados e são apresentado na Tabela 8.

Tabela 8 - Autovetores (Eigenvectors) calculados para os indicadores 1.1 e 2.1.

\begin{tabular}{lr}
\hline Verificadores/indicador & Valor do autovetor \\
\hline 1.1 Implementação dos Instrumentos da PNRH & 0,466 \\
1.1.1 Plano diretor de recursos hídricos aprovado & 0,254 \\
1.1.2 Enquadramento dos corpos d'água aprovado & 0,124 \\
1.1.3 Outorga de direito de uso da água & 0,089 \\
1.1.4 Cobrança pelo uso da água & 0,067 \\
1.1.5 Dados disponíveis no Sistema de Informações & \\
\hline 2.1 Comitê atuante na UPGRH & 0,633 \\
\hline 2.1.1 Comitê de bacia em funcionamento & 0,260 \\
2.1.2 Agência de bacia em funcionamento & 0,106 \\
\hline
\end{tabular}

Fonte-es (2019).

O número principal dos autovetores de cada indicador foi calculado por meio da somatória do produto de cada elemento do autovetor multiplicados pelo total de cada coluna calculado na matriz de julgamentos. $O$ número principal dos autovetores do Indicador 1.1 foi igual a 5,421 e do indicador 2.1 foi 3,055. Por meio desses valores foi possível calcular o IC, sendo que o IC para o indicador 1.1 foi igual a 0,105 e para o indicador 2.1 foi 0,027. Diante desses resultados, a TC do indicador 1.1 foi 0,094 e do indicador 2.1 foi 0,047. Como as taxas são inferiores a $0,1(10 \%)$, foi constatada a coerência da matriz de julgamento de ambos indicadores. O percentual de cumprimento de cada verificador é apresentado na tabela 8 , sendo que 0 cumprimento do Indicador 1.1 foi estimado em $62,45 \%$ e do indicador $2.1 \mathrm{em} 79,69 \%$. Logo, o ICE dos instrumentos de gestão da Política Nacional de Recursos Hídricos no estado de Minas Gerais foi de $71,07 \%$, considerada como "contribuição alta".

Figura 2 - Percentual de cumprimento relativo de cada verificador utilizado para avaliar instrumentos de gestão da Política Nacional de Recursos Hídricos no estado de Minas Gerais em relação ao peso atribuído a cada verificador.

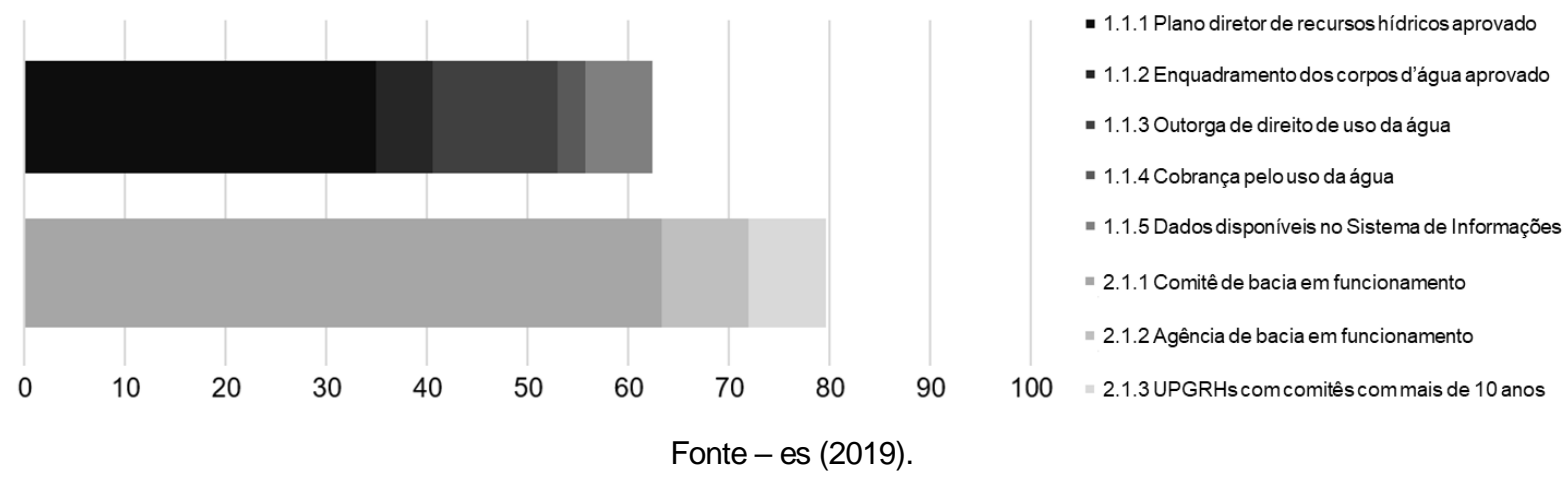

O Método de Multicritérios de Análise de Decisão (MMAD), segundo Boas (2005), é justificado em casos de demasiada complexidade da tomada de decisões, como na avaliação da gestão dos recursos hídricos em 
Minas Gerais, o que justifica a aplicação do mesmo no presente trabalho.

A técnica AHP se mostrou eficaz aos es Shabbir e Ahmad (2016) na avaliação da vulnerabilidade dos recursos hídricos de Rawalpindi e Islamabad, no Paquistão, permitindo estabelecer um sistema de índices e construção de matriz de julgamento. Por meio dessa mesma técnica, Hott et al. (2016) elaboraram um modelo para estimar classes de aptidão ao uso público no Parque Nacional da Serra da Canastra em Minas Gerais, comprovando sua efetividade para apoio à gestão de espaços públicos protegidos. Petrini et al. (2016), também utilizaram a mesma técnica para priorizar políticas públicas voltadas para a agricultura familiar, baseando-se em pesquisas realizadas no município de Ipiranga, no estado do Goiás, concluindo que a metodologia gera resultados confiáveis e que facilitou o processo de tomada de decisão na formulação de políticas públicas. Já Ávila et al. (2018) aplicaram a AHP em um estudo para apoiar o desenvolvimento da Avaliação Ambiental Estratégica da bacia no Rio Grande, no estado de Minas Gerais, possibilitando a análise de diferentes critérios para inferir do grau de conservação da bacia.

\section{CONSIDERAÇÕES FINAIS}

A presente pesquisa revelou que cerca de $71 \%$ dos instrumentos de gestão da Política Nacional de Recursos Hídricos no estado de Minas Gerais foram implementados de maneira eficiente, porém a aprovação do enquadramento dos corpos d'água, a cobrança pelo uso da água e o funcionamento de agências de bacias hidrográficas ainda são insuficientes.

É essencial que todos os instrumentos de gestão de recursos hídricos estejam integrados para se obter um bom planejamento. No entanto, no estado de Minas Gerais, ainda há carências relacionadas, principalmente, ao sistema de informações do estado ser novo (2014) e não estar totalmente alimentado de informações.

A metodologia aplicada no presente trabalho é justificada devido à complexidade que envolve a gestão dos recursos hídricos em Minas Gerais, uma vez que uma multiplicidade de critérios deve ser avaliada. Nesse sentido, o Processo Hierárquico Analítico (AHP) demonstrou-se adequado para a avaliação dos critérios propostos no trabalho, possibilitando, inclusive, a agregação de novos critérios.

\section{AGRADECIMENTOS}

Agradecemos a Coordenação de Aperfeiçoamento de Pessoal de Nível Superior - CAPES, pela concessão de bolsas de estudos.

\section{REFERÊNCIAS}

AITH, F. M. A.; ROTHBARTH, R. O estatuto jurídico das águas no Brasil. Estudos Avançados, São Paulo, v. 29 , n. 84 , p. 163-177, ago. 2015. DOI: 10.1590/S0103-40142015000200011. https://doi.org/10.1590/S0103-40142015000200011

ALENCAR, E. Pesquisa social e monografia. Lavras: Ed. UFLA, 2003. 132 p.

ARRUDA, L. T.; NETO, J. Q. T. Desenvolvimento Sustentável, Prevenção e Precaução: Aplicação no Sistema Jurídico Brasileiro e na Gestão dos Recursos Hídricos. Revista jurídica da FANAP, ano 4, n. 04, jan./jun. 2017. http://187.45.244.77/ojs-2.4.6/index.php/juridica/article/view/67.

ÁVILA, P. A.; MORAS FILHO, L. O.; MENDONÇA, N. P.; BORGES, L. A. C.; GOMIDE, L. R. ACERBI JÚNIOR, F. W. Relevance of strategic environmental assessment to rio grande basin management. Cerne, v. 24, n. 3, p. 225-232, 2018. https://doi.org/10.1590/01047760201824032559

BOAS, C. L. V. Análise da aplicação de métodos multicritérios de apoio à decisão (MMAD) na gestão de recursos hídricos. In: SIMPÓSIO BRASILEIRO DE RECURSOS HÍDRICOS, 16., 2005, João Pessoa. Anais... João Pessoa: ABRH, 2005. p. 1-19.

BRASIL. Lei no 9.433, de 8 de janeiro de 1977. Disponível em: < http://www.planalto.gov.br/ccivil_03/Leis/L9433.htm> Acessado em: 15 de dezembro de 2017.

BRASIL. Constituição (1988). Centro de Documentação e Informação, 446 p. 1988. 
CENTER FOR INTERNATIONAL FORESTRY RESEARCH. Modelo genérico de critérios e indicadores do CIFOR. Manuais: CIFOR, 1999. 59 p. ISBN: 979-8764-46-3.

EDUARDO, E. N. et al. Hydrological simulation as subside for management of surface water resources at the Mortes River Basin. Ciência e Agrotecnologia. Lavras, v. 40, n. 4, p. 390-404, 2016. DOI: https://doi.org/10.1590/1413-70542016404009516

GOMES, A. P. C. Critérios e indicadores de sustentabilidade para o manejo de florestas tropicais. Dissertação (Mestrado em Engenharia Florestal) - Viçosa, UFV. 2000.

HOTT, M. C.; MORAS FILHO, L. O.; FONTES, M. A. L.; PEREIRA, A. A. S.; NOGUEIRA, C. O. G.; CARVALHO, L. M. T.; BORGES, L. A. C.; RESENDE, J. C.; ANTUNES, M. A. H. Public Use and Landscape Analysis in the Serra da Canastra National Park, Brazil: A Geospatial Approach. Natural Resources, v.7, p. 93-101, 2016. DOI: http://dx.doi.org/10.4236/nr.2016.72009

INSTITUTO BRASILEIRO DE GEOGRAFIA E ESTATÍSTICA. Indicadores de desenvolvimento sustentável: Brasil. Rio de Janeiro: IBGE, 2004. Disponível em: <https://ww2.ibge.gov.br/home/geociencias/recursosnaturais/ids/ids_2004.shtm>. Acessado em: 15 dezembro de 2017.

INSTITUTO BRASILEIRO DE GEOGRAFIA E ESTATÍSTICA. Estatísticas por cidade e estado: Minas Gerais. Rio de Janeiro: IBGE, 2018. Disponível em: <https://cidades.ibge.gov.br/brasil/mg/panorama>. Acessado em: 05 julho de 2019.

JUNIOR, D. R. P.; NETTO, O. M. C.; POMPEMAYER, R. S. Análise Multicritério como Instrumento de Gestão de Recursos Hídricos: O Caso das Bacias dos Rios Piracicaba, Capivari e Jundiaí. Revista Brasileira de Recursos Hídricos. Porto Alegre, v. 12, oㅡ 3, jul/set, p.117-127. 2007. https://doi.org/10.21168/rbrh.v12n3.p117-127

KATKO, T. S.; HUKKA, J. J. Social and Economic Importance of Water Services in the Built Environment: Need for More Structured Thinking. Procedia Economics and Finance, v. 21, p. p. 217-223. 2015. DOI: https://doi.org/10.1016/S2212-5671(15)00170-7

LEMOS, R. S.; MAGALHÃES JUNIOR, A. P. Reflexões sobre os critérios de cálculo de vazões outorgáveis em áreas de conflito do estado de Minas Gerais: o caso da Bacia do Ribeirão Ribeiro Bonito. Revista Espinhaço, UFVJM, v. 4, n. 2, p. 4-12, 2015. ISSN 2317-0611.

MARíN, O. A. H. et al. Panorama da gestão dos recursos hídricos no estado do rio grande do Norte, Brasil. Revista Formação, v. 1, n. 23, p. 248-273, 2016. ISSN: 2178-7298.

MENDOZA, G. A. et al. Guidelines for applying multi-criteria analysis to de assessment of criteria and indicators. Jakarta: Center for International Forestry Research, 1999. Disponível em: <http://www.cifor.org/acm/methods/toolbox9.html>. Acessado em: 01 janeiro de 2018.

MINAS GERAIS. Decreto oㅜ 47.343, de 23 de janeiro de 2018. Disponível em:

$<$ http://jornal.iof.mg.gov.br/xmlui/handle/123456789/194466?paginaCorrente=05\&posicaoPagCorrente=19 4470\&linkBase=http\%3A\%2F\%2Fjornal.iof.mg.gov.br\%3A80\%2Fxmlui\%2Fhandle\%2F123456789\%2F\&to talPaginas $=44 \&$ paginaDestino $=01$ \&indice $=0>$. Acessado em: 25 de janeiro de 2018 .

PETRINI, M. A. et al. Using an analytic hierarchy process approach to prioritize publicpolicies addressing family farming in Brazil. Land Use Policy, v. 51, p. 85-94. 2016. DOI: https://doi.org/10.1016/..landusepol.2015.10.029

SAATY, T. L. Multicriteria decision making: the analytic hierarchy process. Pittsburgh: RWS Publications, v. 1, 518 p. 1990.

SELLTIZ, C.; WRIGHTSMAN, L. S.; COOK, S. W. Métodos de pesquisa das relações sociais. São Paulo: Herder, 1965. $136 \mathrm{p}$.

SHABBIR, R.; AHMAD, S. S. Water resource vulnerability assessment in Rawalpindi and Islamabad, Pakistan using analytic hierarchy process (AHP). Journal of King Saud University - Science, v. 28, p. 293299. 2016. https://doi.org/10.1016/j.jksus.2015.09.007

TAFT, L.; EVERS, M. A review of current and possible future human-water dynamics in Myanmar's river basins. Hydrological Earth System Science, v. 20, p. 4913-4928, 2016. https://doi.org/10.5194/hess-20$\underline{4913-2016}$ 
VARGAS, R. V. Utilizando a programação multicritério (analytic hierarchy process - ahp) para selecionar e priorizar projetos na gestão de portfólio. In: PMI GLOBAL CONGRESS 2010, Washington. Proceedings... Washington: Pmi Global, 2010. p. 1-22.

WENG, S. Q.; HUANG, G. H.; LI, Y. P. An integrated scenario-based multi-criteria decision support system for water resources management and planning - A case study in the Haihe River Basin. Expert Systems with Applications, v. 37, p. 8242-8254. 2010. DOI: https://doi.org/10.1016/i.eswa.2010.05.061

Recebido em: 15/02/2018

Aceito para publicação em: 26/11/2019 